\title{
El Semáforo de Neumáticos como técnica en la recuperación de cascos para su renovado y reutilización en el transporte de carga vehicular
}

\author{
The Traffic Light of Tyres as a Technique in the Recovery of Helmets for Renewal and Reuse in \\ the Transport of Vehicle Cargo
}

\author{
Arturo Contreras-Juárez ${ }^{a}$, Alfredo J. Mejia-Sosa ${ }^{b}$, Marcelino García-Miguel $^{b}$, Jonatan $^{-}$ \\ Cardenas-Rangel ${ }^{b}$, Rafael Granillo-Macías ${ }^{c}$, Francisco Baños-Islas $^{d}$
}

\begin{abstract}
:
The main challenge in the administration, of road transport, is to control efficiently the costs related to the operation, where after the fuel the highest costs are the tires. Therefore, this article presents the application of the technique of Semaphore of Tyres, which identifies the wear of the tread, establishing a projection of the number of tyres in good condition, the number of tyres to be renewed in the current period and the budget to be considered for the process in order to minimise operating costs in freight transport. It presents a comparative statement in the projection of costs for changing new and renewed tyres.
\end{abstract}

Keywords:

Tyres, Costs, Transport of ground cargo, Traffic lights on tyres

\section{Resumen:}

El principal desafío en la administración, del transporte carretero, es controlar eficientemente los costos relacionados con la operación, donde después del combustible los gastos más altos son los neumáticos. Por lo tanto, este artículo presenta la aplicación de la técnica de Semáforo de Neumáticos, el cual identifica el desgaste de la banda de rodamiento, estableciendo una proyección del número de neumáticos en buen estado, el número de neumáticos que han de ser renovados en el periodo corriente y el presupuesto que ha de considerarse para su proceso a fin de minimizar los costos de operación en el transporte de carga. Presenta un estado comparativo en la proyección de los costos por cambio de neumáticos nuevos y renovados.

\section{Palabras Clave:}

Neumáticos, Costos, Transporte de carga terrestre, Semáforo en neumático

\footnotetext{
a Autor de Correspondencia, Universidad Politécnica de Tlaxcala Región Poniente, https://orcid.org/0000-0002-7192-3596, Email: arturocontreras@uptlaxponiente.edu.mx

b Universidad Politécnica de Tlaxcala Región Poniente, Email: jhiovani.mejia.lot@gmail.com

cUniversidad Politécnica de Tlaxcala Región Poniente, https://orcid.org/0000-0001-6139-4443, Email: marcelinogarcia@uptlaxponiente.edu.mx

d Universidad Politécnica de Tlaxcala Región Poniente, Email: jonatan.cardenas.range10226@gmail.com

e Universidad Autónoma del Estado de Hidalgo, Escuela Superior Ciudad Sahagún https://orcid.org/0000-0002-1015-667X, Email: rafaelgm@uaeh.edu.mx

d Universidad Tecnológica de Tlaxcala. Tlaxcala México, Email: banosislas@uttlaxcala.edu.mx
} 


\section{Introducción}

Los neumáticos son dispositivos mecánicos de alta tecnología hechos de caucho, químicos y acero que cuando son montados en una rueda del automotor proveen la tracción y soportan la carga.1

El neumático integra el único punto de unión entre el vehículo y la superficie del suelo, donde sus principales funciones es soportar el peso del vehículo cuando está detenido y tolerar las sobrecargas dinámicas que se producen entre cada aceleración y frenado., Al mismo tiempo mitiga las irregularidades de la carretera brindando confort y confianza al conductor, pasajeros y/o cuidado de la carga, transmite la potencia del motor y guiar el vehículo entre otras. 2

En México se producen alrededor de 23 millones de neumáticos, sin embargo, expertos en la materia estiman que la demanda actual oscila en 37 millones, donde los productos de hule que más se importan son los neumáticos nuevos de caucho, que contribuyen con $55.8 \%$ del total. De éstos, $60 \%$ son destinados para vehículos ligeros y $29 \%$ a vehículos pesados. 3

En el autotransporte los neumáticos representan el segundo gasto más importante en el costo total de operación solo detrás del combustible. Puede representar entre el $40 \%$ y el $60 \%$ del gasto de mantenimiento vehicular de una flota. Para un vehículo da carga pesada, contar con un neumático en condiciones sanas de operación es fundamental para eficientar su trabajo. 4

Por otra parte, los materiales de los neumáticos, tardan cientos de años en descomponerse al mismo tiempo su estructura física no se compacta generando un volumen de ocupación más amplio. Los neumáticos usados generan contaminación visual, ya que representa una falta de armonía en el ambiente y cerca de 1000 millones de neumáticos llegan al final de su vida útil en todo el mundo. 5

En México se desechan 40 millones de toneladas de neumáticos. Países como Corea del Sur, Estados Unidos y algunos europeos tienen una tasa de recuperación de neumáticos de $85 \%$, pero no sucede lo mismo en países emergentes. En México se reciclan sólo 10\%; el resto termina en vertederos, rellenos sanitarios, ríos y calles, de acuerdo con datos de la Secretaria de Medio Ambiente de México. 6

Los neumáticos que están incorporados a la industria hulera tuvieron una aportación promedio en 2015 del $0.53 \%$ al Producto Interno Bruto (PIB). 7
En México la industria del neumático genera alrededor de 13,961 empleos, entre directos $(8,614)$ e indirectos $(5,077)$, cantidad que ha venido incrementando en los últimos años. De acuerdo a las estadísticas de la Secretaria de Comunicaciones y Transporte, en el 2013 se movieron 45,288,000 de usuarios en transporte ferroviario y 3,450,000,000 en transporte terrestre, lo cual, muestra que el $98.7 \%$ del transporte es terrestre. La cifra anterior demuestra la importancia de este medio de transporte y por ende de los neumáticos. 8

De acuerdo a la empresa Bridgestone, el ahorro que se genera entre un renovado de neumáticos y un neumático nuevo puede ser de hasta un $18 \%$ del costo total en neumáticos al año. 9

Al mismo tiempo el proceso de renovado contribuye al cuidado del medio ambiente ya que datos de la Agencia Norteamericana de Protección al Ambiente (EPA por sus siglas en ingles), la fabricación de un neumático nuevo de camión requiere de 83 litros de crudo; mientras que la fabricación de una banda de rodamiento para un neumático de camión solo requiere de 26 litros de petróleo, así el proceso de renovado ahorra 57 litros de petróleo por cada neumático, donde cada neumático puede ser renovado hasta tres veces generando un mayor rendimiento. 10

De esta forma el ahorro energético asociado al renovado de neumáticos se cuantifica en el aporte de energía y materiales necesarios para la producción de neumáticos renovados, y se ha comparado con los estimados para la producción de neumáticos nuevos. De esta comparación se ha deducido unos valores medios de ahorro considerables que se estiman en un $69.6 \%$ para un neumático tipo de vehículo comercial y en un $44.5 \%$ para un neumático tipo de turismo. 11

Por otra parte la tabla 1. Muestra el ahorro energético entre los materiales requeridos para la fabricación de neumáticos y su proceso de manufactura entre los neumáticos nuevos y renovados identificando los beneficios de ahorro energético del renovado sobre los nuevos en un $69.6 \%$.

Tabla 1. Ahorro energético en la producción de neumáticos.

\begin{tabular}{llll} 
Comerciales & Nuevo & Renovado & Ahorro \\
\hline Materiales & 104.46 & 36.32 & $65.20 \%$ \\
Proceso & 39.69 & 6.55 & 82.1 \\
\hline Total & 141.2 & 42.87 & 69.6
\end{tabular}

Fuente: M. Sánchez (2013). 
Por lo tanto, este artículo pretende a través de la implementación de la técnica de semáforo de neumáticos en el transporte de carga, la recuperación de cascos para su renovación de la banda de rodamiento a fin de minimizar el gasto de mantenimiento y contribuir a la logística verde al disminuir el número de desecho de neumáticos de camión.

Este artículo se divide en 4 partes, la primera muestra una introducción de una forma general que son los neumáticos y su importancia en el transporte carretero, así como su aportación económica y el impacto ambiental que este representa y sus beneficios económicos y ecológicos al ser renovado. La segunda referente a los Materiales y Métodos, nos describe el proceso general del renovado de los neumáticos y las características técnicas de la aplicación del semáforo de neumáticos, la tercera nos muestra los resultados y beneficios económicos, así como el impacto ambiental que este represento por parte de la empresa en estudio, y en la cuarta sección se muestra una conclusión a fin de sensibilizar al lector, las conclusiones y la bibliografía para el desarrollo y justificación del artículo.

\section{Materiales y Métodos}

El renovado de neumáticos es un conjunto de innovadores procesos por medio de los cuales los cascos en buen estado obtienen una extensión de vida útil similar a la que ofrece un neumático nuevo, disminuyendo así costos de mantenimiento y reemplazo.12

El renovado de neumáticos de carga es tan seguro que permite operar a los neumáticos renovados en las mismas condiciones de carga y velocidad de diseño, del neumático de banda original, con los mismos cuidados que se debe dar a estas.13

El proceso de renovado presenta 10 pasos generales 1.Recolección de cascos de las flotas hacia la planta renovadora. 2.- Inspección visual por dentro y fuera del casco marcando todos los daños visibles. 3.- Raspado se retira el diseño original o la banda anterior del casco, quita el hule viejo básicamente. 4.- Excavación retiro del óxido, suciedad, hule suelto y cualquier material extraño que pueda contaminar el proceso. 5.- Reparación elimina cualquier daño identificado durante la inspección inicial, realizan las reparaciones necesarias para ir devolviendo al casco su vida útil. 6.- Se rellenan los daños vacíos para que no exista aire atrapado al interior del neumático. 7.Embandado se extruye una capa de caucho cojín sin curar sobre la superficie raspada y se rellenan todas las cavidades. De este modo la carcasa del neumático está lista para una nueva banda de rodamiento. 8.Encamisado la banda nueva es cubierta con una camisa elástica con el objetivo de aislar el aire y el vapor. 9.Vulcanizado una cámara de curado hace que la capa de caucho cojín en el neumático se vulcanice y adhiera permanentemente al casco la nueva banda de rodamiento. 10.- Revisión final se repite una inspección visual para asegurar que las especificaciones de calidad se cumplan en su totalidad. El proceso de renovado de neumáticos es una opción para reducir costos por kilómetro con toda la seguridad de tener unos neumáticos que seguirán dando su máximo desempeño en el camión en cualquier camino. 14

Un sólo renovado puede minimizar los costos de desgaste del neumático hasta un $17 \%$. Con un segundo y tercer renovado en ese mismo casco, puede reducir sus costos de desgaste del neumático hasta un $29 \%$ si se compara con un casco sin renovar. 15

\section{Técnica Semáforo de Neumáticos}

El semáforo de neumáticos, es una técnica recomendada por los departamentos de ingeniería de diferentes llanteras como Michelin, Bridgestone, Goodyear entre otras, por otra parte esta técnica no cuenta con una orientación procedimental escrita por los especialistas, sin embargo para este caso de estudio la empresa en cuestión permite describir y documentar su aplicación identificando que esta técnica muestra alertas o riesgos en el neumático, detecta de una forma rápida y sencilla los neumáticos que deben ser retirados para poder enviarlos a renovar. Funciona como mantenimiento preventivo para programar las unidades que tengan neumáticos cercanos al punto de retiro.

Para este caso se consideran tres etapas en la aplicación de la técnica de Semáforo de Neumáticos, con una aplicación de riguroso orden ya que de ello depende que la información recabada en cada una de estas etapas sea la correcta, para tomar medidas necesarias que prolongan el tiempo de vida de los neumáticos, reducen los inventarios en el almacén y solo se efectúen paros programados para cambios de neumáticos requeridos y no por condiciones de un mal uso, que son las causas más frecuentes de retiro de neumáticos cuya vida útil solo se aprovecha en un $50 \%$ o menos en algunos casos de acuerdo a la empresa en cuestión.

En la primera etapa se genera un formato para la recuperación de la siguiente información: Identificación del vehículo (No. Económico), posición de montaje del neumático en el vehículo, profundidad de piso o remanente del neumático para este caso la medida está 
dada en 32/vos, marca del neumático, Identificación del neumático si es original o renovada y el diseño de renovado, presión de aire en el neumático identificado en libras por pulgada cuadrada (PSI).

La segunda etapa está dada por la recuperación física de información: Posición del neumático, medida, si es renovada u original, profundidad de piso, presión de aire.

En la tercera etapa corresponde la interpretación del registro de la información de acuerdo a las especificaciones establecidas en la tabla 1 y la toma de decisiones en el número de neumáticos enviados a renovar sujeta al presupuesto por parte de la empresa.

La tabla 2 muestra las características de profundidad de piso o banda de rodamiento en 32/vos que es la unidad de medida estándar en la empresa de estudio, así mismo establecen los parámetros utilizados en el Semáforo de Neumáticos definidos por la empresa en recomendación de la renovadora Vulcanova S.A. de C.V. para garantizar el retiro en tiempo de los neumáticos originales y renovados a fin de ser nuevamente reutilizados a través del proceso de renovado.

Tabla 2. Parámetros del Semáforo de Neumáticos.

\begin{tabular}{cc} 
32/vos & Observaciones \\
\hline $\mathbf{0 - 4}$ & Retirar para renovar \\
\hline $5 \_8$ & Próximo retiro \\
\hline $9+$ & Buenas condiciones \\
\hline 6 & Direcciónales rotar \\
\hline $\mathbf{X}$ & Retiro de servicio \\
\hline
\end{tabular}

Fuente: Elaboración propia de datos obtenidos de la empresa.

La tabla 3. Presenta el semáforo de neumáticos con una muestra de 5 tractos el cual identifica los neumáticos que deben de retirarse para su renovación de la banda de rodamiento.

Tabla 3. Semáforo de Neumáticos.

\begin{tabular}{|c|c|c|c|c|c|c|c|c|c|c|c|}
\hline ن் & $\begin{array}{l}\frac{5}{0} \\
\frac{0}{0} \\
\text { 음 }\end{array}$ & 1 & 2 & 3 & 4 & 5 & 6 & 7 & 8 & 9 & 10 \\
\hline \multirow[b]{2}{*}{ T1 } & 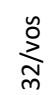 & 9 & 8 & 11 & 11 & 9 & 9 & 4 & 5 & 4 & 4 \\
\hline & $\begin{array}{l}\frac{\pi}{0} \\
\sum^{\pi / 0}\end{array}$ & $\begin{array}{l}0 \\
\dot{j} \\
\dot{\underline{U}}\end{array}$ & $\begin{array}{l}0 \\
\frac{\dot{U}}{\Sigma} \\
\dot{\Sigma}\end{array}$ & 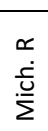 & $\begin{array}{l}\propto \\
\dot{E} \\
\dot{\Sigma}\end{array}$ & $\begin{array}{l}0 \\
\dot{j} \\
\dot{\underline{U}}\end{array}$ & 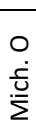 & 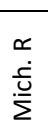 & $\begin{array}{l}\simeq \\
\dot{\underline{J}} \\
\dot{\Sigma}\end{array}$ & $\begin{array}{l}\propto \\
\dot{\delta} \\
\dot{\underline{U}}\end{array}$ & $\begin{array}{l}\propto \\
\dot{S} \\
\dot{\Xi}\end{array}$ \\
\hline
\end{tabular}

\begin{tabular}{|c|c|c|c|c|c|c|c|c|c|c|c|}
\hline & 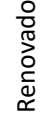 & & & 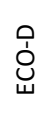 & $\begin{array}{l}\text { o } \\
\text { ঠ્ }\end{array}$ & & & ò̀ & 离 & ò & 엃 \\
\hline & 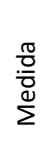 & 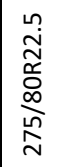 & 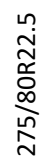 & 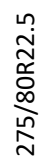 & 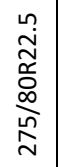 & 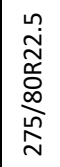 & 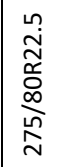 & 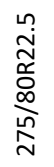 & 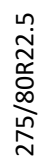 & 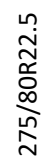 & $\begin{array}{l}\stackrel{n}{N} \\
\underset{N}{\tilde{O}} \\
\stackrel{\infty}{\infty} \\
\stackrel{N}{N}\end{array}$ \\
\hline & PSI & 95 & 105 & 90 & 100 & 100 & 100 & 100 & 85 & 100 & 100 \\
\hline & 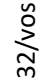 & 12 & 11 & 9 & 13 & 13 & \begin{tabular}{|l|l} 
& 14
\end{tabular} & 13 & 13 & 3 & 3 \\
\hline & 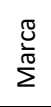 & $\begin{array}{l}0 \\
\frac{\dot{\delta}}{\breve{L}}\end{array}$ & 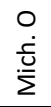 & $\begin{array}{l}\propto \\
\dot{L} \\
\dot{L} \\
\Sigma\end{array}$ & $\begin{array}{l}\propto \\
\dot{L} \\
\dot{\underline{L}} \\
\Sigma\end{array}$ & 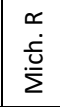 & 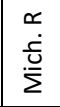 & $\begin{array}{l}\propto \\
\dot{L} \\
\dot{\underline{L}} \\
\dot{\Sigma}\end{array}$ & 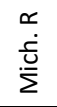 & $\begin{array}{l}0 \\
\frac{\dot{\delta}}{\grave{\Sigma}} \\
\end{array}$ & $\begin{array}{l}0 \\
\dot{j} \\
\dot{\underline{U}}\end{array}$ \\
\hline T2 & 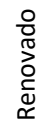 & & & Ò่ & $\begin{array}{l}0 \\
\text { ó } \\
\text { Un }\end{array}$ & 엄 & O̊̀ & $\begin{array}{l}\text { ò } \\
\text { Ou }\end{array}$ & ò̀ & & \\
\hline & $\begin{array}{l}\frac{\pi}{0} \\
\frac{\pi}{0} \\
\sum\end{array}$ & 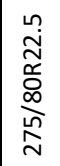 & 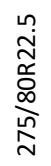 & 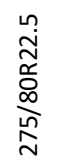 & 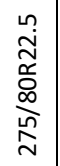 & 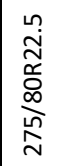 & 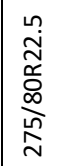 & 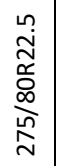 & 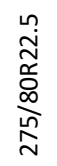 & $\begin{array}{l}\stackrel{n}{N} \\
\underset{\sim}{\tilde{N}} \\
\stackrel{\infty}{\infty} \\
\stackrel{N}{N}\end{array}$ & 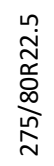 \\
\hline & PSI & 105 & 105 & 100 & 100 & 100 & 100 & 35 & 100 & 100 & 90 \\
\hline & 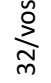 & 7 & 9 & 5 & 5 & 4 & 4 & 14 & 14 & 13 & 13 \\
\hline & $\frac{\substack{0 \\
\frac{\pi}{\pi}}}{\sum}$ & $\begin{array}{l}0 \\
\frac{\dot{\delta}}{\grave{M}}\end{array}$ & 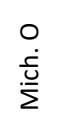 & 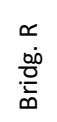 & $\begin{array}{l}\propto \\
\frac{\square}{00} \\
\frac{0}{\bar{L}} \\
\end{array}$ & 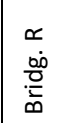 & 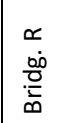 & $\begin{array}{l}0 \\
\dot{\underline{U}} \\
\dot{\underline{\Sigma}}\end{array}$ & $\begin{array}{l}0 \\
\dot{\delta} \\
\dot{\underline{U}}\end{array}$ & $\begin{array}{l}\simeq \\
\dot{\cup} \\
\dot{\infty}\end{array}$ & $\begin{array}{l}\propto \\
\dot{\omega} \\
\dot{\omega}\end{array}$ \\
\hline T3 & 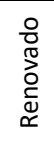 & & & 仓̊̀ & ò & 엄 & 옳 & & & ò่ & 員 \\
\hline & $\begin{array}{l}\frac{\pi}{0} \\
\frac{0}{0} \\
\sum \\
\Sigma\end{array}$ & 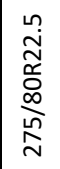 & 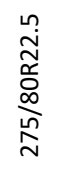 & 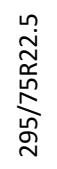 & 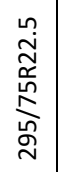 & 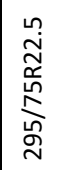 & 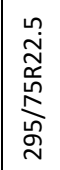 & 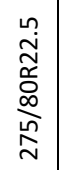 & 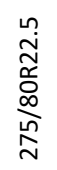 & 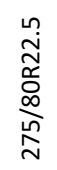 & 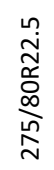 \\
\hline & PSI & 100 & 100 & 95 & 90 & 90 & 100 & 100 & 95 & 100 & 100 \\
\hline & 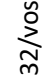 & 6 & 6 & 3 & 5 & 3 & 3 & 8 & 3 & 6 & 6 \\
\hline & 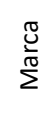 & $\begin{array}{l}0 \\
\dot{s} \\
\stackrel{.}{\Sigma}\end{array}$ & 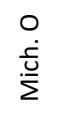 & 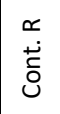 & $\begin{array}{l}\propto \\
\dot{0} \\
\dot{u}\end{array}$ & 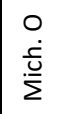 & 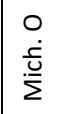 & $\begin{array}{l}\propto \\
\dot{Z} \\
\dot{U}\end{array}$ & $\begin{array}{l}\propto \\
\dot{c} \\
\dot{u}\end{array}$ & $\begin{array}{l}\circ \\
\stackrel{\dot{L}}{\breve{U}} \\
\dot{\Sigma}\end{array}$ & 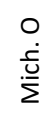 \\
\hline TL & 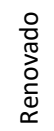 & & & 옳 & ò & & & ò & ò & & \\
\hline & $\begin{array}{l}\frac{\pi}{0} \\
\frac{0}{0} \\
\sum \\
\Sigma\end{array}$ & 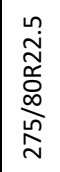 & 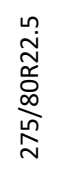 & 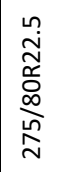 & 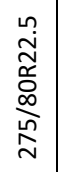 & 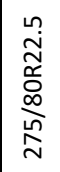 & 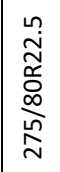 & 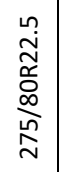 & 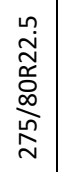 & $\begin{array}{l}\stackrel{n}{\sim} \\
\underset{\sim}{\tilde{N}} \\
\stackrel{\infty}{\infty} \\
\stackrel{\sim}{\sim}\end{array}$ & 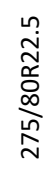 \\
\hline & PSI & 105 & 105 & 100 & 100 & 80 & 95 & 100 & 100 & 90 & 100 \\
\hline
\end{tabular}




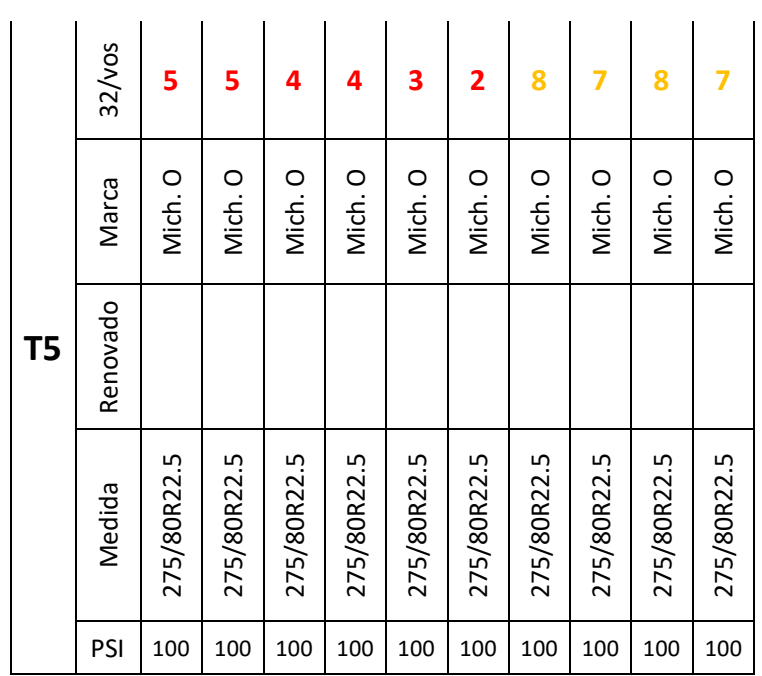

Fuente: Elaboración propia de datos obtenidos en estudio de campo.

Para estimar el porcentaje de ahorro entre neumáticos nuevos y renovados la tabla 4 y 5 presentan una cotización promedio de acuerdo a la medida del neumático, diseño de nuevo y diseño de banda para el renovado que se encuentra en el mercado actual.

Tabla 4. Diseño y costo de renovado de neumáticos.

\begin{tabular}{cll} 
MEDIDA & \multicolumn{1}{c}{ DISEÑO } & \multicolumn{1}{c}{ PRECIO } \\
\hline \multirow{3}{*}{ 11R22.5 5} & T4100 & $\$ 2.180,00$ \\
\cline { 2 - 3 } & FCR-T & $\$ 1.960,00$ \\
\cline { 2 - 3 } 275/80R22.5 & BTR-SA & $\$ 2.500,00$ \\
\cline { 2 - 3 } & ECO-D & $\$ 2.480,00$ \\
\cline { 2 - 3 } & T4100 & $\$ 2.050,00$ \\
\cline { 2 - 3 } & FCR-T & $\$ 1.850,00$ \\
\cline { 2 - 3 } & BTR-SA & $\$ 2.350,00$ \\
\hline \multirow{2}{*}{ 295/75R22.5 5} & $\$ 2.330,00$ \\
\cline { 2 - 3 } & T4100 & $\$ 2.190,00$ \\
\cline { 2 - 3 } & FCR-T & $\$ 1.900,00$ \\
\cline { 2 - 3 } & BTR-SA & $\$ 2.430,00$ \\
\hline \multirow{2}{*}{ 295/80R22.5 5} & $\$ 2.320,00$ \\
\cline { 2 - 3 } & T4100 & $\$ 1.200,00$ \\
\cline { 2 - 3 } & FCR-T & $\$ 1.920,00$ \\
\cline { 2 - 3 } & BTR-SA & $\$ 2.440,00$ \\
\hline & ECO-D & $\$ 2.490,00$ \\
\hline
\end{tabular}

Fuente: Elaboración propia de datos obtenidos de Vulcanova empresa renovadora de neumáticos.

Tabla 5. Costo promedio de los neumáticos originales identificadas en el Semáforo de Neumáticos.

\begin{tabular}{lc} 
Originales & Costo promedio \\
\hline Dirección & $\$ 8.914,00$ \\
\hline
\end{tabular}

\begin{tabular}{ll} 
Tracción & $\$ 12.340,00$ \\
\hline Eje libre & $\$ 11.900,00$ \\
\hline
\end{tabular}

Fuente: Elaboración propia de datos obtenidos de la empresa de transportes.

Para este caso de estudio se presenta una muestra de 5 tractos de entre 15 unidades: 5 Tractos, 5 Remolques y 5 Dollys, Generando un semáforo total de 130 neumáticos.

\section{RESULTADOS}

En la interpretación del Semáforo de Neumáticos se identifica, un total de 7 marcas diferentes en neumáticos utilizados por la empresa de transportes, donde la más utilizada es Michelin con un número total de 70 neumáticos y la de menor uso es la Hankook con 10.

La grafica 1 identifica el número de neumáticos por marca encontrados en el semáforo de neumáticos, a fin de proponer una estandarización al reducir el número de las diferentes marcas para minimizar el riesgo de malos apareamientos entre duales.

Grafica 1. Multimarcas en la muestra del semáforo de Neumáticos.

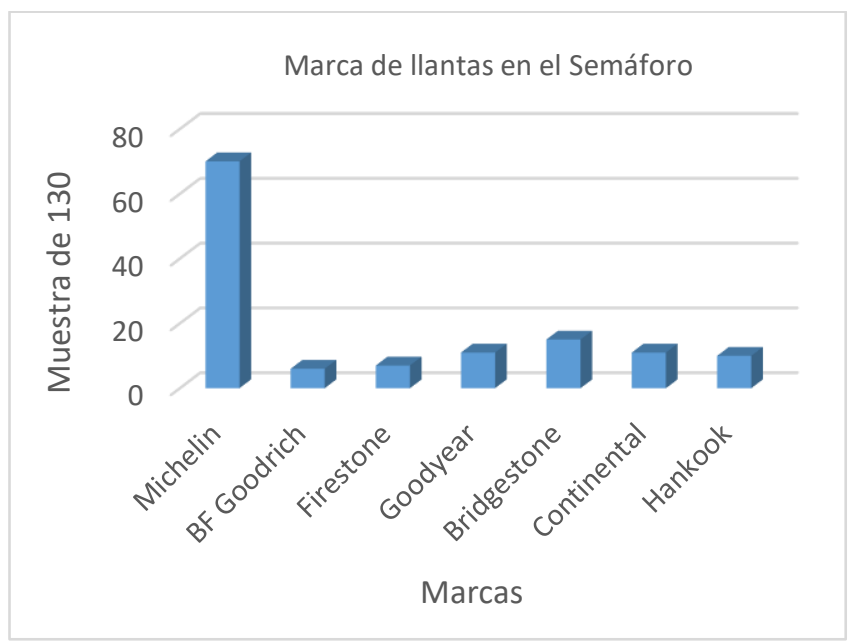

Fuente: Elaboración propia de datos obtenidos de Semáforo de Neumáticos.

Otro dato relevante, la empresa trabaja con renovados encontrando diseños de ECO-D en 22 neumáticos de tracción para tractos, en remolques y Dollys se encontraron diseños de T4100 con 42, FCR-T con 10 y BTR-SA con 4 neumáticos respectivamente.

Del total de neumáticos en el Semáforo se ubicaron 52 neumáticos originales y 78 renovados. La tabla 6 y la gráfica 2 hacen la representación del costo para la empresa. Esta representación está basada en el costo total del neumático nuevo o renovado y su 
proporcionalidad de acuerdo al estado de desgaste actual.

Tabla 6. Costo del Semáforo de Neumáticos.

\begin{tabular}{lccc} 
Neumáticos & No. & Promedio $(\$)$ & Costo total \\
\hline Originales & 52 & $\$ 11.051,00$ & $\$ 574.652,00$ \\
\hline Renovadas & 78 & $\$ 2.162,00$ & $\$ 168.636,00$ \\
\hline Total & 130 & & $\$ 743.288,00$
\end{tabular}

Fuente: Elaboración propia de datos obtenidos del Semáforo de Neumáticos.

Grafica 2. Costo del Semáforo de Neumáticos.

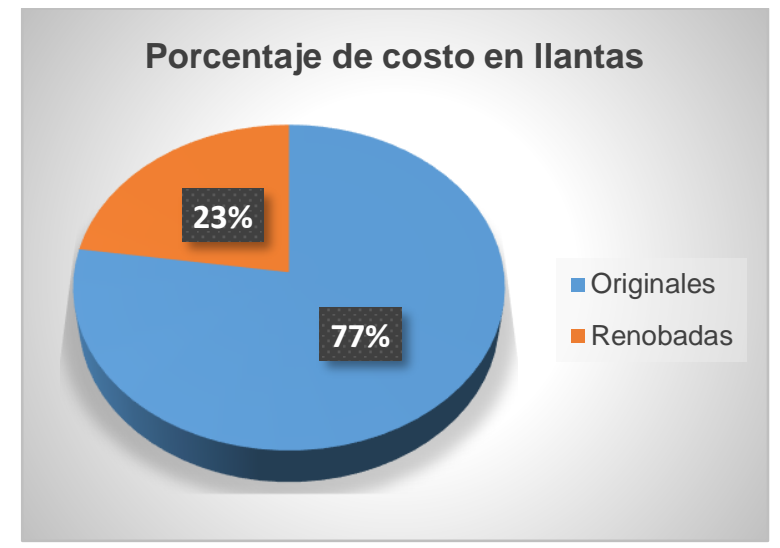

Fuente: Elaboración propia de datos obtenidos del semáforo de neumáticos.

Con respecto a la gráfica 2 se muestra que la inversión en costo de neumáticos originales, superan en gran medida el costo del renovado donde en relación al factor de renovabilidad se entiende que por cada neumático original se tiene 1.5 neumáticos renovados.

La tabla 7 presenta el reporte de Semáforo de Neumáticos el cual indica el número de neumáticos en tiempo de ser retirados para su renovado, así como los neumáticos que hay que retirar de servicio para evitar daños o mantenimientos imprevistos, establece las próximas a renovar apoyando a la elaboración del presupuesto de neumáticos en un próximo periodo a considerar.

Tabla 7. Reporte del Semáforo de Neumáticos.

\begin{tabular}{clc} 
32/vos & Observaciones & No. \\
\hline $\mathbf{X}$ & Retiro de servicio & 4 \\
\hline $0-4$ & Retirar para renovar & 29 \\
\hline $5-8$ & Próximo retiro & 38 \\
\hline $9+$ & Buenas condiciones & 55 \\
\hline
\end{tabular}

\begin{tabular}{cc}
6 Direcciónales rotar & 4 \\
\hline Total & $\mathbf{1 3 0}$
\end{tabular}

Fuente: Elaboración propia de datos obtenidos de Semáforo de Neumáticos.

De esta forma se prevé que de acuerdo al semáforo de neumáticos, 29 de color rojo son prioridad de a ser sustituidos en una primera etapa, considerando que fueran cambiados por neumáticos nuevos tendría un costo promedio de $\$ 320,580.00$ por otra parte si son recuperados los cascos y estas son renovadas se considera un costo promedio de $\$ 62,698.00$, el cual solo representaría el $19.6 \%$ del costo de neumático nuevo.

Por otra parte, los 38 neumáticos de color amarillo son los próximos a retirar en una segunda etapa, considerando una proyección de presupuesto para neumáticos nuevos de $\$ 419,938.00$ o un presupuesto de $\$ 82,156.00$ en caso de recuperar los cascos y ser renovados.

Considerando la opción de renovado de neumáticos se tendría un ahorro de $\$ 595,592.00$ en un periodo de 2 meses y un ahorro de petróleo de 3819 litros.

\section{Conclusiones}

La administración y control de neumáticos representa un área de oportunidad para minimizar los costos en el transporte de carga al reducir los inventarios en cuestión de neumáticos, por lo tanto la técnica de Semáforo de Neumáticos presentada en este artículo pretende generar sistemas de apoyo a las pequeñas y medianas empresas de transporte, que puedan ser representativos en la administración de flotas, así mismo se busca generar un mantenimiento preventivo en los neumáticos que ayuden a maximizar su rendimiento para garantizar una mejor funcionalidad y evitar paros innecesarios por neumáticos en malas condiciones o más aun generar incidentes carreteros por los mismos evitando gastos innecesarios. El conocer información como las condiciones de presión de inflado, marcas de los neumáticos, medidas y profundidad de piso garantiza un mejor servicio del transporte y permite tomar decisiones para hacer correcciones preventivas y evitar que estas sufran desgaste prematuro generando un mayor rendimiento al mismo tiempo que se contribuye a disminuir la contaminación por exceso de neumáticos desechados reflejado en un ahorro de energía. 


\section{Referencias}

[1] INEN. (2012). Neumáticos. Definiciones y clasificación... 1996, de instituto ecuatoriano de normalización sitio web: http://web.ambiente.gob.ec/documents/10179/249439/NTE+2096+Neum $\%$ C3\%A1ticos.pdf/786adf76-cf49-458c-aea9-

92f8af926082;jsessionid=gsJ59KS4gjQWkwwiEjJzXwvD?version=1.0

[2] Michelin. (2019). Encuentra los neumáticos adecuados. 30-08-2019, de Michelin Sitio web: https:/camion.michelin.es/

[3] Castro, G. (2007). Reutilización, reciclado y disposición final de neumáticos. 12-08-2019, de departamento de ingeniería mecánica fiuba sitio web:

https://campus.fi.uba.ar/file.php/295/Material_Complementario/Reutilizaci on_Reciclado_y_Disposicion_final_de_Neumatico.pdf

[4] Alcantar, R.A., Treviño, F.E., \& Martínez, J.L., (2015). Modelo estadístico que permite observar el impacto de los factores que inciden en el rendimiento de combustible. 07/08/2019, de Nova scientia, ISSN: 2007070

[5] Sanz, E. (2019). Cuántos neumáticos se tiran cada año a la basura. Muy Interesante. Recuperado de

https://www.muyinteresante.es/curiosidades/preguntas-respuestas/cuantosneumaticos-se-tiran-cada-ano-a-la-basura-941375271546

[6] Meza, E. (2018). Crean artículos sustentables con llantas usadas. 08-082019, de El Economista Sitio web: https://www.eleconomista.com.mx/empresas/Crean-articulos-sustentablescon-llantas-usadas-20181203-0056.html

[7] Instituto Nacional De Estadística y Geografía (INEGI). (2016). Estadísticas a propósito de la Industria hulera. 15-08-2019, de INEGI Sitio web: http://www.cnih.org.mx/hule 8ago2016 camara.pdf

[8] Manejo responsable de llantas usadas, A.C (2015). Plan de manejo de neumáticos usados de desecho. 18-08-2019, de manejo responsable de llantas usadas, A.C sitio web: http://reciclallantas.org.mx/wpcontent/uploads/2016/02/Plan-de-Manejo-de-Neumaticos-Usados-deDesecho-2015-.pdf

[9] S, M. D. (2014). Renovado reduce $18 \%$ costo anual en llantas. 20-082019, de t21mx sitio web:

http://t21.com.mx/automotriz/2014/03/11/renovado-reduce-18-costoanual-llantas

[10] Potapov, O. (2017). Neumáticos recauchutados: descubre sus pros y sus contras. 21-08-2019, de top concesionarios Sitio web: https://topconcesionarios.com/blog/neumaticos-recauchutados/

[11] Sánchez, M., \& Guzmán, M. (2013) Análisis de la Eficiencia Medioambiental de Recauchutado de Neumáticos Ed. UMH ISBN: 978-8496297-92-0

[12] Bridgestone. (2016). ¿Cómo se renueva una llanta? 23-08-2019, de Bridgestone Sitio web: https://www.bridgestonecomercial.com.mx/es$\underline{\mathrm{mx} / \text { noticias/como-se-renueva-una-llanta }}$

[13] QuimiNet. (2011). Las 6 principales etapas en el renovado de llantas. 25-08-2019, de QuimiNet Sitio web:

https://www.quiminet.com/articulos/las-6-principales-etapas-en-el-

renovado-de-llantas-48116.htm

[14] GALGO. (2019). Proceso de renovado. 29-08-2019, de GALGO Sitio web: http://galgo.com.mx/productos/renovado-de-llantas/proceso-derenovado/

[15] BANDAG. (2019). Te decimos 3 beneficios de renovar tus llantas. 2808-2019, de BANDAG Sitio web:

http://www.bandag.com.mx/Comunicados/comunicado4.html 